\title{
Potencialidades das tecnologias de informação e comunicação para a área das ciências da natureza: uma investigação em
}

\author{
periódicos da área
}

\begin{abstract}
Potentiality of information and communication technologies for natural sciences: an investigation in the journals in the area
\end{abstract}

\author{
Débora Luana Kurz ${ }^{1}$ \\ Everton Bedin²
}

\section{Resumo}

Esta pesquisa tem por objetivo apresentar e refletir sobre uma investigação acerca da utilização das Tecnologias de Informação e Comunicação (TIC) no ensino de Ciências da Natureza no início do processo de escolarização. Para tanto, realizou-se uma revisão bibliográfica para elucidar pesquisas relacionadas a essa temática, assim como identificar como estas são utilizadas no início do processo de escolarização, pontuando vantagens e limitações quanto à utilização destes recursos, averiguando se há a predominância de uma área do conhecimento. Para tal, ao realizar-se um agrupamento dos artigos selecionados em unidades temáticas, com o intuito de propiciar discussões aprofundadas sobre estes, constatou-se que as propostas pedagógicas permeadas pela utilização das TIC propiciam a dinamização dos processos de ensino e aprendizagem, em decorrência de uma série de contribuições, bem como fomentam os processos tanto de interação quanto de diálogo e de colaboração entre os indivíduos envolvidos nesse processo. No entanto, sugere-se a constituição de cursos de formação continuada para professores da área de Ciências da Natureza, com intuito de capacitá-los frente ao uso das tecnologias em sua prática docente.

Palavras-chave: Tecnologias. Ciências da Natureza. Anos Iniciais. Ensinoaprendizagem.

\section{Abstract}

This research aims to present and reflect on research on the use of Information and Communication Technologies (ICT) in the teaching of Natural Sciences at the beginning of the schooling process. To this end, a literature review conducted to elucidate research related to this theme, as well as identify how they are used at the beginning of the schooling process, pointing out advantages and limitations regarding the use of these resources, ascertaining if there is a predominance of an area of knowledge.

\footnotetext{
${ }^{1}$ Mestranda do Programa de Pós-graduação em Ensino de Ciências e Matemática na Universidade Luterana do Brasil/ ULBRA. E-mail: kurz.deboraluana@gmail.com.

${ }^{2}$ Doutorado em Educação em Ciências Quìmica da Vida e Saúde ( Ufsm - Furg) pela Universidade Federal do Rio Grande do Sul, Brasil(2015). Professor Adjunto da Universidade Luterana do Brasil. E-mail: bedin.everton@gmail.com.
}

Interfaces da Educ., Paranaíba, v.10, n.30, p. 199 - 220, 2019

ISSN 2177-7691 
For such, when the selected articles grouped in thematic units, in order to provide in-depth discussions about them, it was found that the pedagogical proposals permeated by the use of ICT provide the dynamization of teaching and learning processes, as a result of a series of contributions, as well as foster the processes of both interaction, dialogue and collaboration between the individuals involved in this process. However, it is suggested the establishment of continuing education courses for teachers in the area of Natural Sciences, in order to enable them in the face of the use of technologies in their teaching practice.

Keywords: Technologies. Natural sciences. Early years. Teaching-learning.

\section{Introdução}

O ensino de Ciências da Natureza para os Anos Iniciais do Ensino Fundamental tem como finalidade promover a aprendizagem dos conhecimentos científicos, os quais corroboram para a compreensão dos fenômenos naturais presentes no entorno dos estudantes. Estes se caracterizam como subsídios para a participação nas tomadas de decisões e transformações, de modo consciente e reflexivo, realizadas em seu contexto, assim como aspectos oriundos dessa intervenção (FABRI; SILVEIRA, 2016). Nessa perspectiva, compreende-se que o ensino de Ciências deve-se vincular a propostas de cunho investigativo, permeadas pelas Tecnologias de Informação e Comunicação (TIC), fomentando o envolvimento do sujeito na construção do conhecimento, em sua curiosidade, senso crítico e problematização, durante os processos de ensino e aprendizagem, transcendendo uma perspectiva de ensino pautada na exposição e memorização dos conteúdos (PINHEIRO, 2014).

Este desenho é importante porque as TIC, "além de passarem a ocupar um espaço significativo na sociedade como mecanismos de entretenimento, progressivamente são utilizadas pelos profissionais da educação como um paradigma técnico aliado aos processos de ensino e aprendizagem" (BEDIN, 2017 , p. 212). Assim, a instituição de ensino, conhecida como um local de formação "ética, científica e tecnológica do aprendiz, não pode desassociar-se do mundo acadêmico que a cerca; logo, precisa fazer parte da realidade tecnológica que lhe confere, multiplicando e qualificando o conhecimento aos estudantes nativos digitais" (BEDIN, 2017, p. 212), principalmente daqueles que se encontram no início do processo de escolarização. 
Neste sentido, entende-se que as tecnologias "funcionam como um meio didático para facilitar os processos de ensinagem, já que possuem fácil manuseio e adesão, permitindo que o aluno encontre seu próprio caminho na ressignificação do conhecimento" (BEDIN; DEL PINO, 2017, p. 70). Segundo os autores, o uso das TIC é essencial "para extrapolar o espaço físico e exceder os muros da escola, garantindo aos educandos a minimização nas barreiras do saber entre professor-aluno e uma forma diferente de ampliação de conteúdos e saberes por meio de pesquisas, ensino e extensão" (BEDIN; DEL PINO, 2017, p. 71).

Desse modo, ressalta-se que a utilização das TIC para a abordagem de fenômenos naturais no ensino de Ciências da Natureza, assim como de objetos das demais áreas do conhecimento, propicia a dinamização dos processos de ensino e aprendizagem, em decorrência de uma série de contribuições, bem como fomenta os processos de interação, diálogo e colaboração entre os indivíduos envolvidos (GALVÃO et al., 2017). Ademais, Bedin e Del Pino (2016, p. 33) ajuízam que as TIC, "quando utilizadas para incrementar as relações entre educadores e educandos, contribuindo para aquisição de conhecimento, possibilidade de auto-expressão e troca de saberes, proporcionam mudanças nos paradigmas atuais da educação".

Integrar as TIC ao ensino é uma forma de proporcionar a instrumentalização docente, em relação a aspectos de como agir e interagir perante o mundo, com base na ética e sob uma perspectiva transformadora (MORAN, 2002; BEDIN, 2015). Em consonância, o Pacto Nacional de Alfabetização na Idade Certa (PNAIC) aponta como objetivo do ensino de Ciências a garantia do direito do estudante vivenciar as potencialidades das TIC no processo de alfabetização científica (BRASIL, 2015). Além disso, o PNAIC ressalta, ainda, que a utilização das TIC no ensino de ciências caracteriza-se como uma abordagem que propicia uma série de possibilidades compostas por descobertas e aprendizagem, proporcionando aos estudantes diferentes ações, tanto de compreender como de aplicar os conhecimentos em suas vivências (LORENZETTI; DELIZOICOV, 2001). 
Afinal, de acordo com Bedin (2015, p. 27), a utilização das TIC emerge para a aprendizagem, sendo necessário que os professores se abram a esses recursos, os quais "fomentam a inovação da ciência posta na sociedade, partindo da premissa de modificar o mundo atual por meio da criação de métodos e práticas educacionais". Assim, de acordo com Bedin, Locatelli e Bedin (2016, p. 279), é necessário que o professor perceba "que as mudanças das metodologias e da qualificação dos processos de ensino e aprendizagem podem prover da inserção das TIC", sendo necessário que os sujeitos assumam "as responsabilidades de seu envolvimento com as TICs e agir de modo reflexivo rumo ao uso destas" (p. 279).

Do mesmo modo, por meio destas, pode-se proporcionar momentos que fomentam ações como observar, analisar, pesquisar, criar, validar hipóteses e socializar em grupo, a fim de promover discussões que beneficiam expressivamente os sujeitos (BRASIL, 2015). Além do mais, as TIC podem ser caracterizadas como fonte de informações, seja como ambiente virtual de aprendizagem, repositório de objetos educacionais ou softwares capazes de elaborar material didático e produtos educacionais (SILVA, 2017). Afinal, segundo Bedin e Del Pino (2016, p. 33), as TIC "provocam em sala de aula, e principalmente na metodologia docente, inúmeras mudanças, uma vez que são capazes de disponibilizar uma gama maior de informações, acarretando no estudante o gosto e o desejo pelo saber".

Nesse contexto, ressalta-se que o potencial pedagógico das TIC, em decorrência do rompimento das fronteiras de uma sala de aula, transcendendo aspectos atrelados ao currículo, fundamentados epistemologicamente e didaticamente, propicia a articulação entre vivências, contemplando os mais diversos espaços do saber (SOBREIRA, 2017; BEDIN, 2015). Entretanto, para uma efetiva articulação entre as propostas pedagógicas e o uso das TIC, é necessário refletir acerca da maneira como estas são utilizadas, visto que não consistem apenas em fonte de informação.

A efetividade de abordagens articuladas ao uso de tecnologias é decorrente das possibilidades propiciadas aos estudantes de observar, Interfaces da Educ., Paranaíba, v.10, n.30, p. 199 - 220, 2019 
internalizar e produzir novos conhecimentos e, portanto, de superar um posicionamento no qual as tecnologias são utilizadas em função de seu caráter informacional, funcional e instrumental. Afinal, segundo Bedin (2017, p. 213), as TIC “apresentam uma multiplicidade de ferramentas de comunicação e trabalho, capazes de fazer com que os estudantes aprendam em meio à comunicação e a interatividade, uma vez que se estabelecem condições de suporte para a dinâmica necessária à colaboração e socialização coletiva”.

Considerando tais premissas, esta pesquisa tem como objetivo refletir e discutir acerca da utilização das TIC nos Anos Iniciais do Ensino Fundamental, com ênfase no ensino de Ciências da Natureza. Do mesmo modo, embasada na literatura disponivel, busca-se destacar as potencialidades e as limitações deste uso, bem como aspectos atrelados ao processo formativo dos professores que atuam no início da escolarização, apontando possiveis lacunas em relação a esta temática, a fim de corroborar tanto para a produção do conhecimento científico quanto à compreensão deste recurso em função de sua utilização no âmbito educacional.

\section{Desenho da Pesquisa}

A revisão bibliográfica caracteriza-se como uma pesquisa de cunho exploratório, a qual propicia a familiarização com a temática em estudo, corroborando para aperfeiçoamento de ideias, bem como proporciona subsídios para descobertas. Portanto, entende-se que esta consiste na primeira etapa para a realização de uma investigação científica, visto que é elaborada a partir da literatura existente, considerando uma linha limítrofe da investigação, sendo necessária a definição dos descritores da pesquisa como banco de dados, palavras-chave, período de publicação das pesquisas, dentre outros critérios estipulados pelo pesquisador. Do mesmo modo, deve ser realizada de modo sistemático, atendendo aos requisitos de uma investigação científica, ou seja, além de apresentar a interpretação das pesquisas selecionadas, precisa contemplar uma análise crítica sobre as mesmas (GIL, 2007; OSTERMANN; MOREIRA, 2016). 
A partir de tais premissas, bem como em consideração as questões mencionadas, determinou-se como tópico desta pesquisa a análise das potencialidades da utilização das TIC nos Anos Iniciais do Ensino Fundamental, conforme especificado na Figura 01. Desse modo, foi possivel elucidar pesquisas relacionadas a essa temática, assim como identificar como estas são utilizadas no início do processo de escolarização, bem como pontuar vantagens e limitações quanto à utilização deste recurso, averiguando se há a predominância de uma área do conhecimento. Além disso, investigou-se aspectos atrelados à formação inicial e continuada dos professores alfabetizadores quanto a quesitos relacionados ao desenvolvimento de competências e habilidades digitais para a utilização destes recursos em sala de aula.

Figura 1 - Esquema das etapas realizadas na revisão bibliográfica.

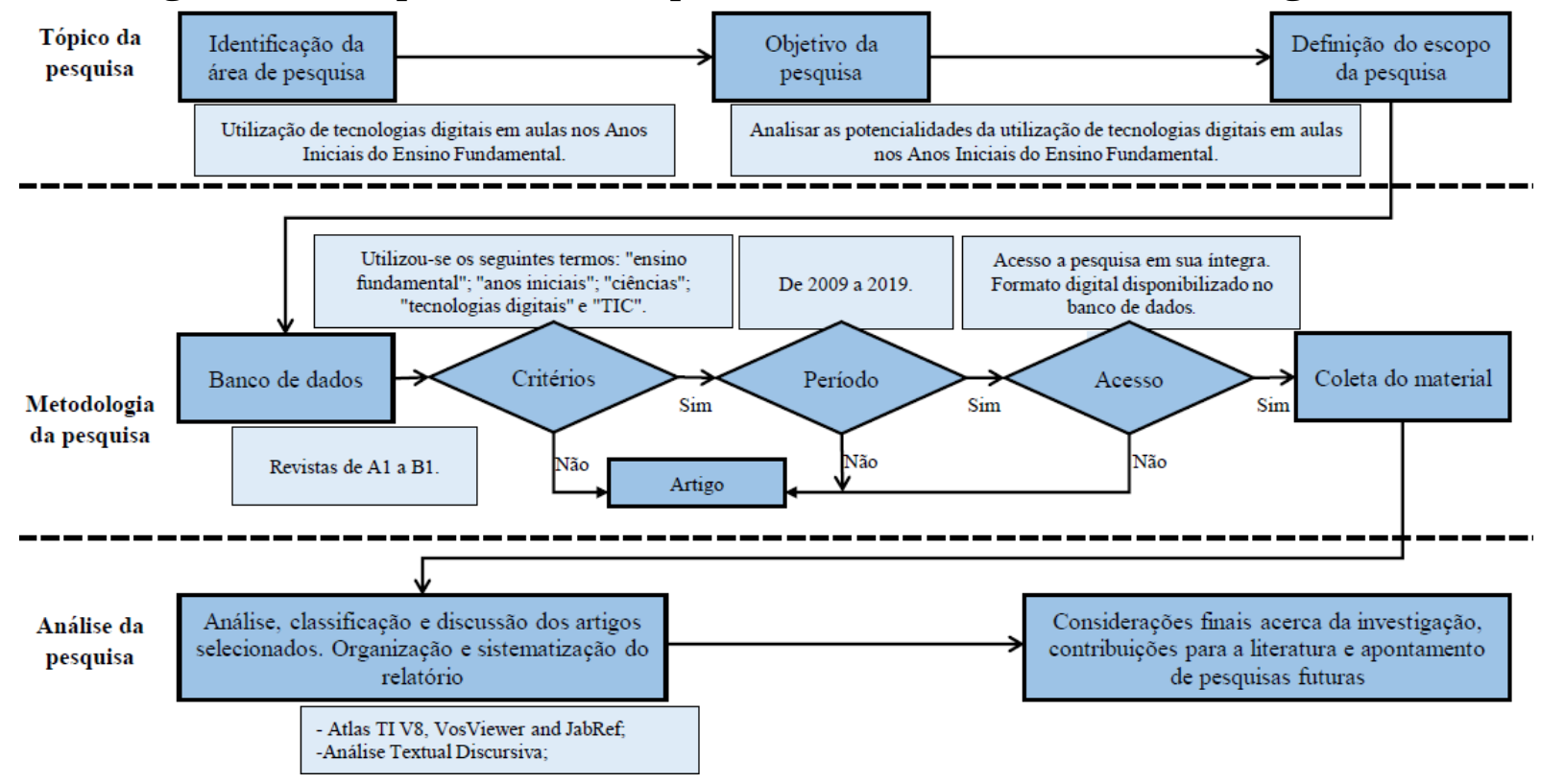

Fonte: Autores da pesquisa, 2019.

Em conformidade com a Figura 1, quanto aos descritores estabelecidos neste estudo, destaca-se o banco de dados. Nesta etapa de busca, considerou-se apenas as revistas classificadas com qualis como A1 à B1, de acordo com sua classificação de periódicos quadriênio de 2013 a 2016, na área de avaliação "ensino", estando atreladas ao título "tecnologias", de modo que possibilitou analisar pesquisas relacionadas à tecnologia da 
educação. Ou seja, artigos que contemplam desde a revisão teórica à relatos de estudo sobre a utilização das TIC nos Anos Iniciais do Ensino Fundamental.

Assim, a partir de tais critérios, selecionou-se as revistas expostas na tabela 1 .

\begin{tabular}{|c|c|c|}
\hline $\mathbf{N}^{\mathbf{0}}$ & Revista & Classificação \\
\hline 1 & Revista Brasileira de Ensino de Ciência e Tecnologia & A2 \\
\hline 2 & Revista Latino-americana de Tecnologia Educativa & A2 \\
\hline 3 & Ciencia, Docencia y Tecnología & B1 \\
\hline 4 & Ensino de Ciências e Tecnologia em Revista & B1 \\
\hline 5 & Revista Novas Tecnologias na Educação & B1 \\
\hline 6 & Revista Ciência e Tecnologia & B1 \\
\hline 7 & Revista de Biotecnologia \& Ciências & B1 \\
\hline 8 & Revista de la Facultad de Ciencia y Tecnología & B1 \\
\hline 9 & Revista Educação \& Tecnologia & B1 \\
\hline 10 & $\begin{array}{l}\text { Revista Iberoamericana de Ciencia Tecnología y Sociedad (En } \\
\text { Linea) }\end{array}$ & B1 \\
\hline 11 & $\begin{array}{l}\text { Revista Iberoamericana de Tecnologia en Educación y Educación } \\
\text { en Tecnología (En Linea) }\end{array}$ & B1 \\
\hline 12 & Revista Tecnologia e Sociedade & B1 \\
\hline 13 & Revista Tecnologia e Sociedade (Online) & B1 \\
\hline 14 & Revista Tecnologias na Educação & B1 \\
\hline 15 & TEAR - Revista de Educação, Ciência e Tecnologia & B1 \\
\hline 16 & Tecnologia Educacional & B1 \\
\hline
\end{tabular}

Fonte: Autores da pesquisa, 2019.

Retomando a análise da Figura 1, a partir da identificação das revistas, iniciou-se a busca dos artigos por meio dos seguintes termos: "Ensino Fundamental I"; "Anos Iniciais"; "Letramento digital"; "Tecnologias digitais", "TIC", "años iniciales", "tecnologias digitales", "alfabetización digital" e "educación primaria", as quais deviam obrigatoriamente constar no título, resumo, palavras-chave, termos indexados e texto completo. Assim, foram considerados somente os estudos realizados em um período de 2009 a Interfaces da Educ., Paranaíba, v.10, n.30, p. 199 - 220, 2019 
2019, os quais se encontram disponíveis no formato digital, com o intuito de realizar uma varredura de pesquisas publicadas recentemente, se caracterizando como pertinentes para o tópico sobre investigação.

Dando prosseguimento às descrições das etapas, de acordo com a Figura 1, têm-se a análise do material coletado, a qual ocorreu por meio da aferição com base na Análise Textual Discursiva - ATD - (MORAES; GALIAZZI, 2013). Atentou-se a realização das quatro etapas específicas no método de análise qualitativa, as quais consistem no processo de unitarização (leitura cautelosa das pesquisas), categorização a partir das relações estabelecidas (acarretando em um novo emergente), o processo de auto-organização (emersão de novas compreensões referentes ao tópico em estudo). Para esta etapa, também se utilizou como recurso de análise o software Atlas.ti.

\section{Resultados e Discussão}

A partir dos descritores estabelecidos nesta pesquisa, selecionou-se 95 artigos atrelados ao tópico de investigação, dentre uma totalidade de 12 revistas que apresentaram resultados perante a busca. Nessa perspectiva, conforme supracitado na metodologia para análise de dados, realizou-se as etapas destacadas na ATD por Moraes e Galiazzi (2013), a fim de evidenciar o caráter narrativo das mesmas. Desse modo, nas respectivas unidades adiante, evidencia-se o posicionamento e a concepção de diferentes autores frente à utilização das TIC nos processos de ensino e aprendizagem nos Anos Iniciais do Ensino Fundamental. Além disto, estabelece-se relações entre estes posicionamentos para identificar lacunas das pesquisas, a fim de pontuar aspectos que ainda necessitam ser investigados.

Nessa perspectiva, a partir da análise dos estudos selecionados no Atlas.ti, elaborou-se a nuvem de palavras (Figura 2), com o intuito de identificar o caráter inicial destas pesquisas. Nesta, salienta-se aspectos atrelados ao uso das TIC como recursos que corroboram nos processos de ensino e aprendizagem, com ênfase a jogos e pesquisas, assim como particularidades relacionadas à formação de professores, em função de sua prática docente e a forma na qual são utilizados em suas atividades. 
Entretanto, ressalta-se que a Figura 2 se caracteriza com uma ideia inicial dos estudos desenvolvidos, os quais serão posteriormente discutidos e refletidos a partir de pequenas especificidades e particularidades.

Figura 2 - Nuvem de palavras.

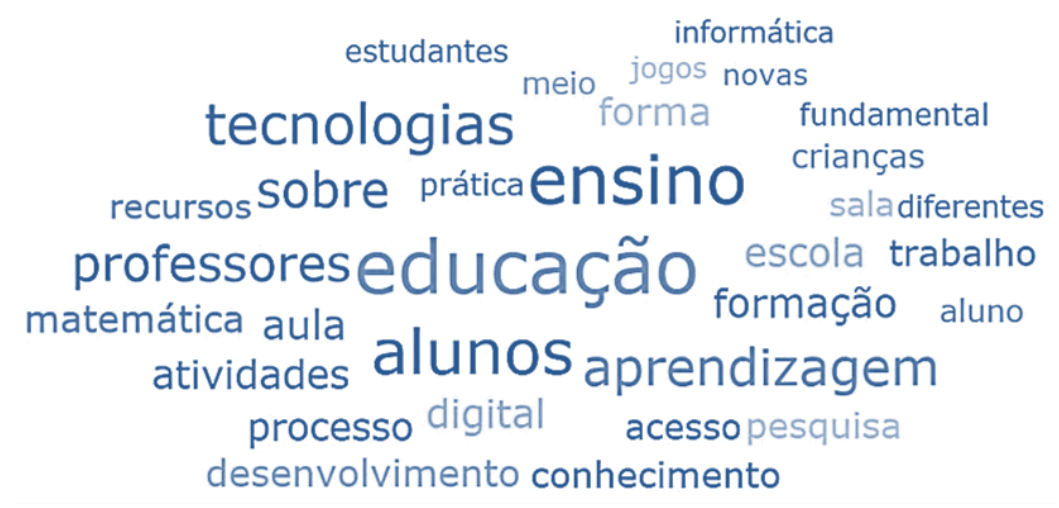

Fonte: Autores da pesquisa, 2019.

Observando-se a nuvem elaborada, percebe-se que esta coincide com as unidades de discussão (Figura 3) propostas a partir da análise qualitativa da ATD (MORAES; GALIAZZI, 2013). Estas foram intituladas como "Desdobramentos das TIC", "Formação de professores para uso das TIC", com exceção da unidade "TIC no ensino de Ciências da Natureza", a qual consiste no foco dessa pesquisa. Este desenho é importante porque a nuvem de palavras é elaborada em detrimento da sua representatividade, o que significa que há poucas pesquisas desenvolvidas nesta área do conhecimento para os Anos Iniciais do Ensino Fundamental, diferentemente do que ocorre em Matemática, como se representa na Figura 2.

Figura 3 - Unidades de discussão. 


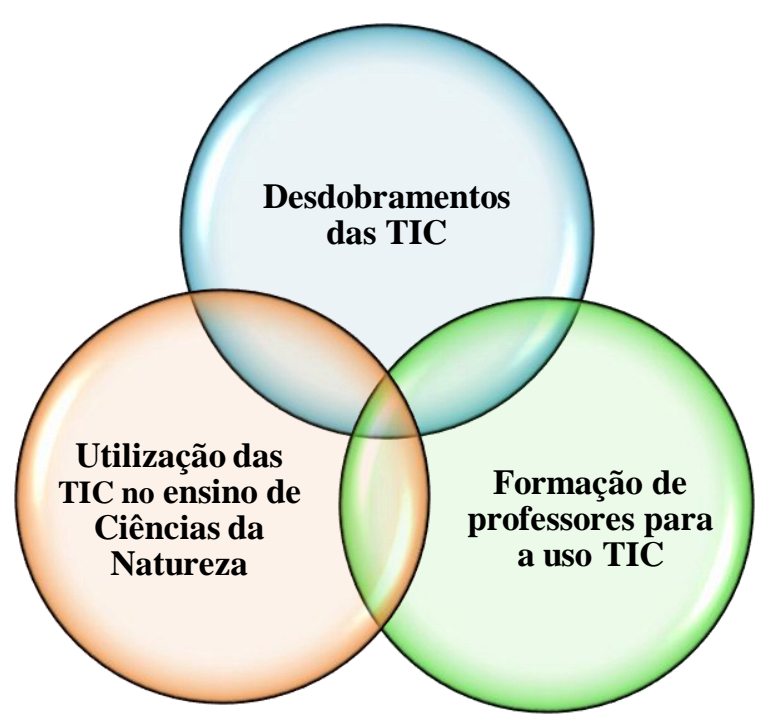

Fonte: Autores da pesquisa, 2019.

Portanto, na sequência, são apresentadas, por meio de uma discussão, as particularidades e as especificidades oriundas das pesquisas correspondentes à determinada unidade temática, as quais emergiram como método qualitativo da Análise Textual Discursiva.

\section{Desdobramentos das tecnologias digitais}

O uso educacional das TIC nos primeiros niveis de ensino contribui para o desenvolvimento de uma série de competências e habilidades, em função da apropriação destes recursos, de modo que o estudante seja capaz de refletir acerca dos conceitos estudados e, partir destes, analisar e testar suas hipóteses, corroborando para a compreensão de novos conteúdos, bem como apropriação de outras estratégias (SOBREIRA, 2017). Não diferente, Papert (2008) complementa que além de corroborar para a construção do conhecimento, o uso destas possibilita diferentes maneiras de pensar e aprender; logo, propiciar-se-á a formação integral do estudante para o exercício pleno da cidadania.

Nessa perspectiva, as propostas pedagógicas permeadas pela utilização das TIC propiciam diferentes abordagens para um mesmo tópico sobre investigação, bem como podem ser adaptadas para diferentes áreas do conhecimento. Por meio desta, é possivel promover o envolvimento dos estudantes nesse processo, corroborando para o desenvolvimento de 
competências e habilidades, aperfeiçoando sua capacidade de resolução de problemas por meio da tomada de decisão e de seu protagonismo no processo de construção do conhecimento. Nesse sentido, destaca-se, a partir da literatura analisada, algumas das possibilidades de abordagens das TIC em consonância com as áreas de conhecimento, as quais foram desenvolvidas nos Anos Iniciais do Ensino Fundamental.

$\mathrm{Na}$ área das Linguagens destacam-se principalmente a literatura infantil, as hiperhistórias e as narrativas, as quais são elaboradas a partir de uma série de recursos, tais como a ferramenta Storybird, assim como são utilizadas no processo de letramento. Ademais, como se pontua a utilização de softwares, como o Menino Curioso e o Tux Paint, destaca-se o acesso a páginas que contemplam atividades educativas, para potencializar o processo de alfabetização. Alguns autores ainda ressaltam a utilização de jogos e o uso de vídeos como facilitadores do processo de apropriação da leitura e da escrita (PEREIRA, 2009; MARIA; MONTOVANO; SARMENTO, 2010; FERREIRA; REHFELDT; SILVA, 2017; SANTOS; FURTADO, 2017; DRAGO; MORAES; COSTA, 2018; LIMA; FRANCO; BERTAGNOLLI, 2018).

$\mathrm{Na}$ área das Ciências Humanas, encontram-se pesquisas que sugerem uma gama de possibilidades para a utilização das TIC. Estas contemplam desde a aplicação de um objeto educacional que possui como temática as "Regiões do Brasil, além de tarefas na Webquest acerca dos pontos turísticos de uma dada cidade, ao desenvolvimento do pensamento espacial a partir da utilização do recurso ROODA (RIBEIRO; ALCALDE; BEHAR, 2010; RADAELLI; FRUET, 2011; CARVALHO et al., 2018).

Em relação à informática, em especial à abordagem dos conceitos de Robótica Educacional, encontra-se na literatura uma série de possibilidades, objetivando a desenvolver o raciocínio dos estudantes, em função da realização de atividades lúdicas. Nesse contexto, estas contemplam desde a utilização da plataforma Code.org para o ensino de programação, como uso de kits Topobo, até pesquisas que se objetivam introduzir os fundamentos da computação, sem a utilização de recursos tecnológicos. (SANTIN; SILVA; BOTELHO; 2012; SCAICO, 2012; CALEGARI; SANTOS; POZZEBON; FRIGO 
2015; GLIZT et al., 2017; KAMINSKI; BOSCARIOLI, 2019; VIVASFERNANDEZ; SÁEZ-LÓPEZ, 2019).

A Matemática se caracteriza como à área do conhecimento que possui o maior número de pesquisas desenvolvidas, contemplando a utilização das TIC desde o início do processo de escolarização. Nessa perspectiva, os estudos apresentam uma série de abordagens que corroboram para a construção do conhecimento, as quais se referem à utilização de vídeos em diferentes tópicos de estudos relacionados ao ensino de Matemática, o uso de recursos tecnológicos como, por exemplo, o PoliKalc, o qual foi desenvolvido com a finalidade de contemplar cálculos aritméticos, visando a compreensão das operações básicas (adição, subtração, multiplicação e divisão). A abordagem de um conteúdo seja em relação à sequência numérica, às operações matemáticas, às frações, os números decimais e geometria, por meio da utilização de tablets, foram alvos de pesquisa, também. (LEANDRO et al., 2016; BORBA; OECHSLER, 2018; CRUZ; BACELLAR; QUARTIERI, 2018; MARTINS; BASSO, 2018).

Ainda, em relação a esta área do conhecimento, ressalta-se a manipulação de dispositivos touchscreen, como recurso para os processos de ensino e aprendizagem em matemática, mais especificamente no ensino de geometria por meio de softwares, sendo eles Geometric Constructer e Sketchometry, e a sugestão de elaboração de Jogos no Scratch numa perspectiva da Modelagem Matemática. Em relação à utilização de demais jogos, como o Lego, trabalhos foram desenvolvidos com o intuito de desenvolver propostas pedagógicas que propiciam o envolvimento dos estudantes neste processo, fomentando a curiosidade e a criticidade destes em relação ao objeto de estudo. (BAIRRAL; ASSIS; SILVA, 2015; KAMINSKI; BOSCARIOLI, 2018; MONZON; BASSO, 2018; VITAL; MENDONÇA, 2018).

\section{Utilização das tecnologias digitais no ensino de Ciências da Natureza}

De acordo com a Base Nacional Comum Curricular - BNCC - (2018), o ensino de Ciências da Natureza tem como objetivo desenvolver as competências e as habilidades necessárias para que o indivíduo seja capaz de compreender o mundo, bem como transformá-lo. Apesar de as pesquisas 
acerca da utilização das TIC no ensino de Ciências no início do processo de escolarização serem incipiente, alguns autores como Rosa et al. (2019) destacam que a utilização de recursos digitais permeados a propostas didáticas corroboram para o processo de alfabetização científica, em decorrência da aproximação entre Ciência, Tecnologia e Sociedade (CTS). Em consonância, ressaltam que as tecnologias devem ser compreendidas como recursos facilitadores, enquanto que os professores são caracterizados como mediadores desse processo.

Mediante tais premissas, destaca-se o posicionamento de Machado et al. (2019), o qual reflete sobre as potencialidades do App Inventor para o ensino de ciências desde os ciclos iniciais, uma vez que consiste em um recurso promissor para o desenvolvimento de aplicativos de cunho educacional para a plataforma Android. Como exemplos desta proposta, têmse o EcoÁgua e a Trilha ecológica, os quais estão relacionados à Educação Ambiental, fomentando a integração e a colaboração entre os sujeitos envolvidos na proposta. Nesse sentido, como destaca Rosa et al. (2019), a utilização das TIC atende as demandas do que almeja-se desenvolver, as quais pontuam-se desde a adoção de propostas diversificadas, flexibilização do plano de ensino, disponibilização de materiais dinâmicos à atualizações dos mesmos.

Melques et al. (2011), em consonância com Tavares, Bertagnolli e Amaral (2016), apontam as estratégias de ensino em Ciências embasadas nos objetos educacionais, uma vez que estes propiciam o enriquecimento do ambiente de aprendizagem potencializando a construção do conhecimento. Um objeto educacional pode consistir em uma atividade experimental, um jogo lúdico ou até mesmo simulações, no qual o estudante possa relacioná-lo com o objeto de estudo, promovendo feedbacks para propiciar a compreensão do fenômeno, além de verificar hipóteses, sendo uma possibilidade de desenvolver o raciocínio crítico e lógico. Desse modo, por meio da utilização de objetos educacionais, é possivel interagir com as teorias científicas subjacentes, as quais não poderiam ser verificadas sem a observação direta. 
Nesta teia, destaca-se o trabalho de Skalee et al. (2017), pois estes realizaram uma investigação acerca da implantação de um jogo educacional, "Fredi no Mundo da Reciclagem", o qual foi elaborado por meio de storyboard, permitindo a organização e a representação dos elementos que compõem o jogo em um projeto gráfico da aplicação. Este ressalta a importância da reciclagem, bem como aspectos em relação à preservação do meio ambiente, em um viés lúdico e dinâmico.

Do mesmo modo, os autores argumentam em relação a suas potencialidades em função de que os aplicativos e os jogos permeados pelas TIC se caracterizam como instrumentos lúdico-didáticos, pois promovem o desenvolvimento de habilidades, fomentam a criatividade e espontaneidade e potencializam o processo de construção do conhecimento, fenômeno que também é abordado em estudos de Mombach (2013), Roma e Lopes (2016) e Brasero e Valencia (2018).

Nessa perspectiva, em relação à diversificação das atividades apontada pelos autores supracitados, Vestena, Scremin e Canto-Dorow (2016) apontam a literatura infantil, enquanto Freitas e Barin (2014) abordam acerca da utilização de histórias em quadrinhos em formato digital, ambos acerca do ensino de Ciências no Ensino Fundamental I. Por meio destas possibilidades didáticas, pode-se promover o acesso a diferentes fontes de informação e estruturas de argumentação, as quais transcendem o repertório sociocientífico de um livro didático. Além disso, é sábio que, da mesma forma que apontam a incorporação das TIC à luz das histórias em quadrinho, estas possibilitam aulas mais interessantes e pertinentes, com ênfase em múltiplos aspectos, visando à colaboração entre os sujeitos envolvidos (BEDIN; DEL PINO, 2017; MACHADO et al., 2019).

Ainda, destaca-se uma série de potencialidades em relação à utilização das TIC, como propostas por Ferreira, Rehfeldt e Silva (2017), acerca do uso de vídeos nas aulas de ciências, bem como as possibilidades educacionais de espaços de criação e prototipação, conhecidos como FabLab, dentre outras, destacado por Borges (2015). Nesse sentido, evidencia-se que há uma abordagem pedagogicamente diversificada atrelada aos recursos digitais, apresentando um respectivo objeto de estudo, mediante diferentes Interfaces da Educ., Paranaíba, v.10, n.30, p. 199 - 220, 2019 
perspectivas, favorecendo os processos de ensino e aprendizagem. No entanto, é imprescindivel proporcionar condições para a realização de propostas pedagógicas que contemplam estes recursos, principalmente no que tange à formação de professores.

\section{Formação de professores para a utilização das tecnologias digitais}

As tecnologias educacionais atreladas a interfaces digitais proporcionam tanto aos docentes quanto aos estudantes à possibilidade de potencializar os processos de ensino e aprendizagem, tendo como finalidade a construção do conhecimento de forma significativa (MORAN, 2013; BEDIN; DEL PINO, 2016). Em consonância, Moura (2017) ressalta que a utilização de recursos por meio de dispositivos multiplataforma, mediados no âmbito educacional, corrobora para a imersão do aluno à aprendizagem, em decorrência da apropriação da linguagem das redes de informação e comunicação. Nessa perspectiva, é necessário que o professor desenvolva as aptidões necessárias para realizar abordagens adequadas a partir da utilização destes recursos, com o intuito de sistematizar a gama de conhecimentos, além de emergir nesse processo.

Em relação à formação inicial e continuada de professores, Dias e Junior (2016), assim como Cruz e colaboradores (2018), ressaltam que, para a efetiva incorporação das TIC no processo educativo, faz-se necessária implantação de projetos e politicas públicas que visam garantir, além da infraestrutura necessária, aspectos atrelados ao processo de reconfiguração socioeducativo, bem como da formação inicial e continuada dos agentes responsáveis por essa transformação. Em decorrência de tais premissas, é necessário (re)pensar o currículo dos cursos de licenciatura, especialmente no que tange a formação de professores para área de Ciências da Natureza; logo, sugere-se a adesão de cursos que promovam o desenvolvimento de competências, habilidades e atitudes digitais, de modo que os docentes sintam-se aptos a utilizar os recursos tecnológicos em suas práticas pedagógicas (MACHADO et al., 2019). 
Nessa perspectiva de (re)pensar a formação docente, Zaccaron (2012), Lotthammer e colaboradores (2017), em suas pesquisas, apontam a Educação a Distância (EaD) como uma possibilidade de formação continuada, de modo a suprir a carência no que tange formação inicial de pedagogos, a qual, em conformidade com Aguiar e Basso (2018), não propicia uma abordagem significativa quanto a inclusão das TIC no âmbito educacional. Assim, é necessário transcender o uso instrumental das TIC por meio de estratégias que corroboram para o desenvolvimento de novas formas de pensar e aprender (LEONEL et al. 2019)

No entanto, uma série de adversidades evidencia o quão labiríntico consiste o processo de integração das TIC na educação. Professores se deparam com diferentes desafios, uma vez que necessitam contemplar curricular e pedagogicamente alunos que apresentam um nivel significativo de apropriação frente ao uso das tecnologias e outros que vivem à margem desse processo, além de instituições que possuem infraestrutura adequada, enquanto outros possuem espaços precários, bem como recursos mínimos (DIAS; JUNIOR, 2016). Neste sentido, questiona-se: como constituir este aluno perante este universo de informações, a fim de que consiga posicionarse criticamente frente ao uso das TIC como mecanismo de crescimento pessoal, cultural, educacional e social à luz do ensino das Ciências da Natureza?

\section{Considerações Finais}

A partir dos desdobramentos desta pesquisa, evidencia-se a necessidade do desenvolvimento de pesquisas relacionadas ao ensino de Ciências da Natureza atreladas ao uso das TIC, em função de seu potencial educativo. Não diferente, também é necessário investigar aspectos oriundos da formação inicial e continuada de professores, em relação ao modo de como estes fazem uso das TIC em suas práticas pedagógicas. Ademais, devese pensar em estratégias a serem desenvolvidas nos Anos Iniciais do Ensino Fundamental no intuito de propiciar a articulação entre os conhecimentos prévios dos alunos, em consonância com as possibilidades derivadas da 
integração das TIC, corroborando para o processo de construção e ressignificação do conhecimento.

Assim, ao desenrolar das investigações, contatou-se um número significativo de pesquisas à luz das TIC relacionadas ao ensino de Matemática, podendo esta propiciar subsídios de como efetivar a integração das TIC no ensino de Ciências da Natureza, bem como das demais áreas do conhecimento. Este processo é importante na medida em que se compreende que as atividades se fundamentam em elaboração de jogos e vídeos, assim como o uso de recursos digitais como tablet, celular e computador para o acesso de páginas e softwares educativos, as quais podem ser adaptadas para o ensino de Ciências em função dos objetivos pedagógicos estabelecidos para determinada atividade.

Por fim, salienta-se que, além de corroborar para os processos de ensino e aprendizagem, as TIC propiciam uma nova perspectiva em relação ao pensar e aprender ao fazer e dizer pedagogicamente. De outra forma, as TIC se caracterizam como aspectos fundamentais para ensino de Ciências em detrimento da busca por uma compreensão concisa de mundo, bem como de modo a atender as demandas da sociedade. Todavia, somente será viável à formação do aluno se esta ocorrer desde o início do processo de escolarização, sendo desafiado a observar, analisar, propor e avaliar hipóteses, de modo que seja capaz de arquitetar e buscar soluções para situações-problema. Para tanto, a ação docente, com base na utilização das TIC, é essencial ao decorrer desta construção.

\section{Referências}

AGUIAR, M. B.; BASSO, M. V. A. Investigação sobre as visões de um grupo de professoras acerca do uso das tecnologias em sala de aula para ensino de matemática: um olhar para a formação das pedagogas e as formas de utilização da tecnologia em seu fazer docente. RENOTE, v. 16, n. 2, 2018.

BAIRRAL, M. A.; DE ASSIS, A. R.; DA SILVA, B. C. C. Uma matemática na ponta dos dedos com dispositivos touchscreen. Revista Brasileira de Ensino de Ciência e Tecnologia, v. 8, n. 4, 2015.

BEDIN, E. A emersão da interdisciplinaridade no ensino médio politécnico: relações que se estabelecem de forma colaborativa na qualificação dos Interfaces da Educ., Paranaíba, v.10, n.30, p. 199 - 220, 2019 
processos de ensino e aprendizagem à luz das tecnologias de informação e comunicação. 2015. 512 f. Tese (Tese de Doutorado) - Programa de Pós Graduação em Ciências: Química da Vida e Saúde, Universidade Federal do Rio Grande do Sul, Porto Alegre, RS, 2015.

BEDIN, E. Aprendizagem Colaborativa, Troca de Saberes e Redes Sociais: tríade na Educação Básica. Revista Brasileira de Ensino de Ciência e Tecnologia, v. 10, n. 2, 2017.

BEDIN, E.; DEL PINO, J. C. Tecnologias no Ensino de Química: Uma Avaliação Neurocientífica para os Processos de Ensino e Aprendizagem. Revista Debates em Ensino de Quimica, v. 2, n. 1, p. 31-40, 2016.

BEDIN, E.; DEL PINO, J. C. Atividade Interdisciplinar de Cunho Tecnológico na Educação Básica/Activity Interdisciplinary of Form Technological in the Basic Education. Revista FSA (Centro Universitário Santo Agostinho), v. 14, n. 2, p. 68-85, 2017.

BEDIN, E.; LOCATELLI, A.; BEDIN, R. J. Tecnologia e Educação: Estado da Arte no paisagismo do SENID. Revista Brasileira de Ensino de Ciência e Tecnologia, v. 9, n. 3, 2017.

BORBA, M. C.; OECHSLER, V. Tecnologias na educação: o uso dos vídeos em sala de aula. Revista Brasileira de Ensino de Ciência e Tecnologia, v. 11, n. 2, 2018.

BORGES, K. et al. Possibilidades e desafios de um Espaço Maker com objetivos educacionais. Tecnologia Educacional, v. 1, p. 22-32, 2015.

BRASERO, T. C.; VALENCIA, L. P. S. EnseñAPP: Aplicación Educativa de Realidad Aumentada para el Primer ciclo de Educación Primaria. Revista Iberoamericana de Tecnología en Educación y Educación en Tecnología, n. 21, p. e01, 2018.

BRASIL. Ministério da Educação. Secretaria de Educação Básica. Para saber mais sobre o Pacto Nacional de Alfabetização na Idade Certa (PNAIC). Brasília, 2015.

BRASIL. Base Nacional Comum Curricular (BNCC). Educação é a Base. Brasília, MEC/CONSED/UNDIME, 2018.

CALEGARI, P.; SANTOS, T. N. dos; POZZEBON, E.; FRIGO, L. B. Utilizando A Robótica Para O Ensino De Lógica Computacional Com Crianças Do Ensino Fundamental. Novas Tecnologias na Educação, v.13, n.2, 2015.

CARVALHO, D; BUENO, C. J. S.; PEDRO, K. M.; SILVA, E. G. Estudo sobre eficácia da aplicação de um objeto de aprendizagem com alunos do ensino 
fundamental. Revista Brasileira de Ensino de Ciência e Tecnologia, v. 11, n. $1,2018$.

CRUZ, R. P. da; BACELLAR, T. M.; QUARTIERI, M. T. Uso educacional de tablets para ensinar matemática nos anos iniciais- Formação continuada. Revista Tecnologias na Educação, v.18, n.1, 2018.

DIAS, C. F. B.; JUNIOR, G. S. Ensino de estatística e tecnologias da informação e comunicação: entre a docência e o desenvolvimento de recursos tecnológicos. Revista Brasileira de Ensino de Ciência e Tecnologia, v. 9, n. 1, 2016.

DRAGO, V. S. R.; MORAES, C. F. B.; COSTA, C. S. A tecnologia nos anos iniciais: novas possibilidades de aprendizagem a partir do gênero contos de fadas. Revista Tecnologias na Educação. 2018.

FABRI, F.; SILVEIRA, R. M. C. F. O ensino de ciências nos anos iniciais do ensino fundamental sob a ótica CTS: uma proposta de trabalho diante dos artefatos tecnológicos que norteiam o cotidiano dos alunos. Investigações em Ensino de Ciências, v. 18, n. 1, p. 77-105, 2016.

FERREIRA, M. S.; REHFELDT, M. J. H.; SILVA, J. S. da. Possibilidades para o uso pedagógico do vídeo no ciclo de alfabetização. 2017.

FREITAS, K. O.; BARIN, C. S. Formação de professores dos Anos Iniciais do Ensino Fundamental: potencialidades e desafios do uso pedagógico das TIC. RENOTE, v. 12, n. 2, 2014.

GALVÃO, R. M.; MARTINS, N.; FRANCO, S. A. P. Artefatos digitais e leitura crítica: formação para a atuação docente. Tecnologia Educacional [on line], Rio de Janeiro, n. 216, p. 33-41, 2017.

GIL, A. C. Como elaborar projetos de pesquisa. 4. ed. São Paulo: Atlas, 2007.

GLIZT, F. R. O. et al. O pensamento computacional nos anos iniciais do ensino fundamental. RENOTE, v. 15, n. 2. 2017.

KAMINSKI, M. R.; BOSCARIOLI, C. Criação de jogos digitais na perspectiva de introdução à Modelagem Matemática nos anos iniciais. Revista Thema, v. 15 , n. 4, p. 1538-1548, 2018.

KAMINSKI, M. R.; BOSCARIOLI, C. Uso Do Ambiente Code.Org Para Ensino De Programação No Ensino Fundamental I - Uma Experiência No Desafio Hora Do Código. Ensino de Ciências e Tecnologia em Revista. 2019.

LEANDRO, E. G.; BATISTA, A. J.L.; IZÁ, S. E.; OLIVEIRA, A. C. O Processo de Criação de um Software para o Ensino e a Aprendizagem de Cálculos 
Aritméticos no Ensino Fundamental. Revista Brasileira de Ensino de Ciência e Tecnologia, v. 9, n. 2, 2016.

LEONEL, A. A. et al. A formação de professores na perspectiva da mídia educação. Ensino de Ciências e Tecnologia em Revista, vol. 9, n. 1, 2019.

LIMA, R. S.; FRANCO, M. H. I.; BERTAGNOLLI, S. C. Jogo educacional digital: Alfabetização na sala de recursos multifuncionais. \# Tear: Revista de Educação, Ciência e Tecnologia, v. 7, n. 1, 2018.

LOTTHAMMER, K. S. et al. Integração de tecnologias digitais na educação: desenvolvimento de MOOC para formação continuada de docentes. Revista Tecnologias na Educação. Ano 09. Vol.23. 2017.

MACHADO, E. F.; DA SILVA, S. C. R.; BASNIAK, M. I.; MIQUELIN, A. F. APP Inventor: da autoria dos professores à atividades inovadoras no ensino de ciências. Revista Brasileira de Ensino de Ciência e Tecnologia, v. 12, n.1, 2019.

MARIA, S. A. A.; MANTOVANI, A. M.; SARMENTO, D. F. Hiperhistória para auxiliar a criança do $2^{\circ}$ ano do ensino fundamental em seu processo de ortografização. RENOTE, v. 7, n. 1. 2009.

MARTINS, E. F.; BASSO, M. V. A. Concepção de Objetos Digitais de Aprendizagem para Combinatória nos Anos Iniciais. RENOTE, v. 16, n. 1. 2018.

MELQUES, P. M. et al. O Ensino De Ciências Naturais Nas Séries Iniciais Do Ensino Fundamental: Uma Estratégia De Ensino Utilizando Objetos Educacionais. RENOTE, v. 9, n. 2, 2011.

MOMBACH, C. O uso do computador na sala de aula de lingua estrangeira: desafios e propostas. EntreVer-Revista das Licenciaturas, v. 3, n. 5, p. 25-40, 2013.

MONZON, L. W.; DE AZEVEDO BASSO, M. V. Prospecção de Pesquisas sobre o uso de Tecnologias Digitais para o Desenvolvimento do Pensamento Geométrico Espacial. RENOTE, v. 16, n. 1. 2018

MORAN, J. M. Novas Tecnologias e Mediação Pedagógica. Editora Papirus, $21^{\mathrm{a}} \mathrm{Ed}, 2013$.

MOURA, R. A. T. Estudo de viabilidade técnica e pedagógica de aprendizagem por dispositivos multiplataforma. Tecnologia Educacional [on line], Rio de Janeiro, n. 216, p. 72-83, 2017.

OSTERMANN, F.; MOREIRA, M. A. Uma revisão bibliográfica sobre a área de pesquisa Física Moderna e Contemporânea no Ensino Médio. Investigações em ensino de ciências, v. 5, n. 1, p. 23-48, 2016. 
PEREIRA, L. L. Softwares educativos: uma proposta de recurso pedagógico para o trabalho de reforço das habilidades de leitura e escrita com alunos dos anos iniciais. 2009.

PINHEIRO, M. R. G. Desafios e perspectivas na inclusão das TIC's como instrumento didático no ensino de conceitos científicos nas séries iniciais do ensino fundamental. Mestrado Profissional em Ensino de Ciências Instituição de Ensino: UNIVERSIDADE ESTADUAL DE RORAIMA, Boa Vista Biblioteca Depositária: Biblioteca Central da Universidade Estadual de Roraima, 2014.

RADAELLI, M. R. R.; FRUET, F. S. O. Processo ensino-aprendizagem e interação entre alunos e professores potencializados pelas Tecnologias da Informação e da Comunicação. RENOTE, v. 9, n. 1. 2011.

RIBEIRO, A. C. R.; ALCALDE, B. F. K.; BEHAR, P. A. Explorando a interatividade no Planeta ROODA 2.0. RENOTE, v. 8, n. 3. 2010.

ROMA, N. S. S. de; LOPES, M. C. Estratégias de ensino-aprendizagem com base em tecnologias digitais: uma análise com professores e coordenadores pedagógicos dos anos iniciais. RENOTE, v. 14, n. 2. 2016

ROSA, C. T. W.; DARROZ, L. M.; MINOSSO, F. B. Alfabetização científica e ensino de ciências nos anos iniciais: concepções e ações dos professores. Revista Brasileira de Ensino de Ciência e Tecnologia, v. 12, n. 1, 2019.

SANTIN, M. M.; SILVA, J. A. da; BOTELHO, S. S. C. Artefatos educacionais com memória cinética Topobo: uma abordagem para o currículo dos anos iniciais. 2012.

SANTOS, D. C. P.; FURTADO, C. C. Recursos Tecnológicos na Literatura Infantil. Revista Tecnologias na Educação, ano 9, n. 18, p. 1-15, $2017 .$.

SCAICO, P. D. et al. Um relato de experiências de estagiários da licenciatura em computaçao com o ensino de computaçao para crianças. RENOTE, v. 10, n. 3, 2012.

SILVA, J. B. Politicas de formação continuada aos professores dos anos iniciais de mato grosso para o uso pedagógico das tecnologias digitais no ensino de ciências. Mestrado em Ensino de Ciências e Matemática, Instituição de ensino: Universidade do Estado de Mato Grosso, Barra do Bugres Biblioteca Depositária: UNEMAT, 2017.

SKALEE, A. A. et al. Fredi no Mundo da Reciclagem: Jogo Educacional Digital para Conscientização da Importância da Reciclagem. RENOTE, v. 15, n. 1, 2017. 
SOBREIRA, E. S. R. Tecnologias digitais no ensino de ciências para crianças: autoria e interações em uma proposta educativa explorando o tema energia. Mestrado em Multiunidades em Ensino de Ciências e Matemática Instituição de Ensino: Universidade Estadual de Campinas, Campinas Biblioteca Depositária: Biblioteca Central da Unicamp, 2017.

TAVARES, M. R. N.; BERTAGNOLLI, S. C.; AMARAL, J.C. S. R. Elaboração de um repositório para a disponibilização de objetos de aprendizagem com foco nos anos iniciais do ensino fundamental. \# Tear: Revista de Educação, Ciência e Tecnologia, v. 5, n. 1, 2016.

VESTENA, R. F.; SCREMIN, G.; CANTO-DOROW, T. S. Ensino de Ciências e histórias infantis: uma proposta para os anos iniciais. \# Tear: Revista de Educação, Ciência e Tecnologia, v. 5, n. 1, 2016.

VITAL, J. E.; DE MENDONÇA, P. C. Jogos na sala de aula: como eles podem ajudar a desenvolver habilidades e conhecimentos matemáticos. ASSOCIE$S E \grave{A} A B T$, p. 86. 2018.

VIVAS-FERNANDEZ, L.; SÁEZ-LÓPEZ, J. M.. Integración de la robótica educativa en Educación Primaria. Revista Latinoamericana de Tecnología Educativa-RELATEC, v. 18, n. 1, p. 107-129, 2019.

ZACCARON, A. B. M. et al. Uso Pedagógico das Tecnologias Digitais: do Fazer ao Compreender. RENOTE, v. 10, n. 3. 2012. 